\title{
2019 novel coronavirus (COVID-19) pneumonia: CT manifestations and pattern of evolution in 110 patients in Jiangxi, China
}

\author{
Jie Zhan ${ }^{1,2} \cdot$ Haijun $\mathrm{Li}^{1,2} \cdot$ Honghui $\mathrm{Yu}^{1,2} \cdot{\text { Xiaochen } \mathrm{Liu}^{3} \cdot \text { Xianjun Zeng }}^{1,2} \cdot$ Dechang Peng ${ }^{1,2}$ (D) $\cdot$ Wei Zhang ${ }^{4}$
}

Received: 29 April 2020 / Revised: 9 August 2020 / Accepted: 18 August 2020 / Published online: 27 August 2020

(C) European Society of Radiology 2020

\begin{abstract}
Objectives To elucidate the CT manifestations and patterns of evolution in patients with COVID-19 pneumonia.

Methods This is a retrospective review of CT scans of 110 patients. All 110 patients had initial and second CT scans, 60 of 110 patients had three serial CT scans, and 17 of 60 patients had four serial CT scans. Numerous characteristics of pulmonary and extrapulmonary abnormalities and recognizable patterns of evolution were evaluated.

Results Of the 110 initial CT scans, ground-glass opacities without consolidation $(65.4 \%)$ were more common than a consolidation or mixed pattern. The most common findings were subpleural involvement (77.2\%), multifocal involvement (80.7\%), and bilateral involvement (67.3\%). Three serial CT scans of 60 patients showed four patterns of CT evolution: type 1 showing relatively high CT scores on initial CT (averaged 4 days after symptom onset), with mild progression and improvement on follow-up CT scans (25\%); type 2 with progression of CT findings from initial CT to first follow-up CT (averaged 9 days after symptom onset) with subsequent improvement on second follow-up CT (averaged 13 days after symptom onset, $61.7 \%$ ); type 3 with no CT changes (5.0\%); and type 4 pattern was similar to type 2 but with a more prolonged course and more severe CT findings $(8.3 \%)$.

Conclusions Predominant findings at initial CT scans were bilateral multifocal subpleural GGO. The most commonly shown evolution pattern was type 2: progression of disease with increased extent and density of opacities on first follow-up CT followed by improvement on second follow-up CT.

Key Points

- Predominant findings at initial CT in patients with COVID-19 infection are bilateral multifocal subpleural ground-glass opacities.

- Ill-defined patchy or nodular opacities were most commonly observed on CT scans with the right lower lobe most commonly involved.

- The most commonly shown evolution pattern on chest CT was type 2: progression of CT findings from initial CT to first followup CT with subsequent improvement on second follow-up CT.
\end{abstract}

Keywords Coronavirus · Infections · COVID-19 · Pneumonia · Tomography, X-ray

\section{Abbreviations}

COVID-19 Coronavirus disease 2019

CT Computed tomography

GGO Ground-glass opacities

Jie Zhan and Haijun Li contributed equally to this work.

Dechang Peng pengdcdoctor@163.com

$\triangle$ Wei Zhang zhangweiliuxin@163.com

1 Department of Radiology, the First Affiliated Hospital of Nanchang University, 17 Yongwai Zheng Street, Donghu District, Nanchang City 330006, Jiangxi, China

\section{Introduction}

In December 2019, a series of respiratory disease cases of unknown etiology were reported in Wuhan, Hubei, China

2 Jiangxi Province Medical Imaging Research Institute, Nanchang 330006, China

3 Department of Radiology, University of Toledo Medical Center, 3000 Arlington Ave, Toledo, OH, USA

4 Department of Respiratory Medicine, the First Affiliated Hospital of Nanchang University, 17 Yongwai Zheng Street, Donghu District, Nanchang City 330006, Jiangxi, China 
[1]. On January 7, 2020, Chinese health authorities confirmed that these were associated with a novel coronavirus, now termed COVID-19 [2]. Common symptoms of patients with COVID-19 infection included fever, fatigue, shortness of breath, and dry cough [3]. Current epidemiologic data indicate that human-to-human transmission of COVID-19 was occurring $[4,5]$. The disease quickly spreads across China and beyond, and as of Apr 29, 2020, a total of 3,018,681 confirmed COVID-19 cases have been reported worldwide with 207,973 deaths [6].

Chest computed tomography (CT) examination plays an important role in diagnosis and monitoring of disease progress especially during the early stage of the pandemic when testing was limited and results were slow to come back. Subsequently, CT scans have also been used to follow up response to medical treatment and to evaluate progression or changes in clinical symptoms. Numerous chest CT features of COVID-19 pneumonia have been described in recent studies [7-9]; however, the patterns of evolution have been less clearly described. More recently, Pan et al investigated the time course of lung changes on chest CT during recovery from COVID-19 pneumonia, and concluded that lung abnormalities on chest CT showed greatest severity approximately 10 days after initial onset of symptoms [10]. Due to the crucial role of CT images in diagnosis, it is imperative for radiologists to be aware of the CT manifestations and pattern of evolution of this global health emergency. The objective of this study is to investigate the chest CT manifestations and pattern of evolution in patients with COVID-19 infection.

\section{Materials and methods}

\section{Subjects}

Between Jan 21, 2020, and Feb 5, 2020, chest CT and clinical records for patients with real-time reverse-transcriptasepolymerase chain reaction (rRT-PCR)-confirmed COVID-19 infection were reviewed retrospectively. For patients with serial CT scans, our last day of follow-up CT was Feb 9, 2020. This study was approved by the Human Research Ethics Committee of the First Affiliated Hospital of Nanchang University; informed consent was waived.

\section{Selection criteria}

At the start of the COVID-19 pandemic, viral testing was limited and the results were slow to come back, hence chest CT was used as the tool for initial diagnosis. For patients hospitalized with COVID-19 pneumonia, follow-up CT scans were used to assess response to medical treatment and/or interventions. The selection criterion for chest $\mathrm{CT}$ was that the patient presented with cough, shortness of breath, and fever.
The selection criterion for the follow-up CT scans was if patients' symptoms changed or progressed. Each subsequent CT scan was performed consecutively for the individual patient.

\section{Imaging techniques}

CT scans were obtained with the patients in the supine position, and scanning was performed at end inspiration. Thinsection CT was performed using one of the following CT scanners: 16-CT Emotion (Siemens Healthcare), or 256-CT Brilliance (Philips Healthcare). The protocol used was as follows: (Siemens Healthcare) $130 \mathrm{kV}, 30-150 \mathrm{mAs}, 8-\mathrm{mm}$ slice thickness; (Philips Healthcare) $120 \mathrm{kV}, 100-120 \mathrm{mAs}$, 5-mm slice thickness. Reconstruction section thickness, $1 \mathrm{~mm}$. All CT scans for each patient were performed with the same CT scanner.

\section{Image analysis}

The initial and serial follow-up CT during treatment were retrospectively reviewed in consensus by two fellowshiptrained thoracic radiologists (D.C. and J.Z.). The images were viewed on lung (window width, $1600 \mathrm{HU}$; window level, $-600 \mathrm{HU}$ ) and mediastinal (window width, $350 \mathrm{HU}$; window level, $60 \mathrm{HU}$ ) windows. The axial CT images were analyzed but multiplanar reformats were available for use should the readers choose to do so.

The CT images were assessed for pulmonary parenchymal abnormalities, including (1) location: the left lung (upper lobe, lower lobe), right lung (upper lobe, middle lobe, lower lobe); (2) distribution: subpleural, random, peribronchovascular; (3) extent of involvement: assessed by visually estimating the percentage of pneumonia involvement in each lobe [7]; (4) morphology: patchy, lobar, segmental, irregular, or nodular; (5) density: ground-glass opacities (GGO), consolidation, mixed pattern of GGO and consolidation; (6) internal structure: crazy-paving pattern, interlobular septal thickening, vascular thickening, air bronchogram, and bronchial wall thickening; (7) edge: well-defined or ill-defined; (8) evolution over time: for the follow-up chest CT, recognizable pattern of change was evaluated by comparing the overall percentage of lung involvement for each patient on serial chest CT. The CT scans were also assessed for extrapulmonary manifestations, including mediastinal lymphadenopathy, pleural effusion, and pleural thickening.

The anatomic distribution was noted as being predominantly subpleural if abnormality involved mainly the peripheral one-third of the lung, random if abnormality involved without predilection for subpleural or central regions, or being peribronchovascular if the abnormality involved along the bronchovascular bundles. GGO were defined as increased lung density without obscuration of the underlying vessels 
[11]. Consolidation was defined as increased lung density with obscuration of the underlying vessels. Crazy-paving pattern was defined as thickened interlobular septa and intralobular lines with ground-glass opacification [12]. Each of the five lung lobes was assigned a score that was based on the following: score $0,0 \%$ involvement; score 1, 1-25\% involvement; score 2, 26-50\% involvement; score 3, 51-75\% involvement; and score 4, 76-100\% involvement. Summation of scores provided overall lung involvement (range of possible scores, 0-20) [7, 13].

Data analysis of the CT evolution pattern was performed based on the stages of COVID-19 pneumonia established by Zhou et al (progression stage from 1 to 7 days, advanced plateau stage from 8 to 14 days, and absorption stage $>14$ days) [14]. In this study, subjects were divided into 4 groups based on the time to peak: group 1 with total CT lung involvement peaking within 7 days; group 2 with total CT lung involvement peaking from days 8 to 14 ; group 3 with total CT lung involvement peaking after day 14; group 4 for patients with asymptomatic infection or with CT scores less than 1.

\section{Statistical analysis}

Statistical analyses were performed using IBM SPSS Statistics Software (version 21; IBM). Quantitative data were presented

Table 1 Clinical characteristics of 110 patients with COVID-19 infection

$\begin{array}{lr}\text { Gender } & \\ \text { Male } & 67(60.9 \%) \\ \text { Female } & 43(39.1 \%) \\ \text { Age (years) } & 43.8 \pm 13.4(18-80) \\ \text { Exposure history } & \\ \text { Living in or traveling to Wuhan within 14 days } & 46(41.8 \%) \\ \text { Exposure to infected patient } & 48(43.6 \%) \\ \text { Unknown exposure } & 16(14.6 \%) \\ \text { Symptoms } & \\ \text { Fever } & 102(92.7 \%) \\ \text { Cough } & 71(64.5 \%) \\ \text { Shortness of breath } & 5(4.5 \%) \\ \text { Sore throat } & 5(4.5 \%) \\ \text { Diarrhea } & 3(2.7 \%) \\ \text { Nausea and vomiting } & 2(1.8 \%) \\ \text { Fear of cold } & 2(1.8 \%) \\ \text { Headache } & 2(1.8 \%) \\ \text { Rhinobyon } & 1(0.9 \%) \\ \text { Asymptomatic } & 2(1.8 \%) \\ \text { Time from symptom onset to performing initial CT } & 4.43 \pm 3.54(0-15) \\ \text { (days) } & \end{array}$

Quantitative data were presented as mean \pm standard deviation (minimum-maximum), while the counting data were presented as percentage of the total
Table 2 Initial chest CT findings of 110 patients with COVID-19 infection

\begin{tabular}{|c|c|}
\hline No involvement & $9(8.2 \%)$ \\
\hline Unilateral lung & $27(24.5 \%)$ \\
\hline Bilateral lung & $74(67.3 \%)$ \\
\hline \multicolumn{2}{|l|}{ Number of lobes affected } \\
\hline 0 & $9(8.2 \%)$ \\
\hline 1 & $21(19.1 \%)$ \\
\hline 2 & $11(10.0 \%)$ \\
\hline 3 & $9(8.2 \%)$ \\
\hline 4 & $18(16.4 \%)$ \\
\hline 5 & $42(38.2 \%)$ \\
\hline \multicolumn{2}{|l|}{ Frequency of lobar involvement } \\
\hline Left upper lobe & $64(63.4 \%)$ \\
\hline Left lower lobe & $72(71.3 \%)$ \\
\hline Right upper lobe & $68(67.3 \%)$ \\
\hline Right middle lobe & $60(59.4 \%)$ \\
\hline Right lower lobe & $87(86.1 \%)$ \\
\hline \multicolumn{2}{|l|}{ Density feature } \\
\hline No involvement & $9(8.2 \%)$ \\
\hline Only GGO & $72(65.4 \%)$ \\
\hline Only consolidation & $6(5.5 \%)$ \\
\hline Both GGO and consolidation & $23(20.9 \%)$ \\
\hline Either GGO or consolidation & $101(91.8 \%)$ \\
\hline \multicolumn{2}{|l|}{ Distribution feature } \\
\hline Subpleural & $65(64.4 \%)$ \\
\hline Random & $11(10.9 \%)$ \\
\hline Peribronchovascular & $12(11.9 \%)$ \\
\hline Subpleural and peribronchovascular & $13(12.9 \%)$ \\
\hline \multicolumn{2}{|l|}{ Morphology } \\
\hline Nodular & $48(47.5 \%)$ \\
\hline Lobar or segmental & $17(16.8 \%)$ \\
\hline Patchy & $70(69.3 \%)$ \\
\hline Irregular & $18(17.8 \%)$ \\
\hline \multicolumn{2}{|l|}{ Margins } \\
\hline Ill-defined & $85(84.2 \%)$ \\
\hline Well-defined & $16(15.8 \%)$ \\
\hline \multicolumn{2}{|l|}{ Signs } \\
\hline Crazy-paving pattern & $37(36.6 \%)$ \\
\hline Vascular enlargement & $43(42.6 \%)$ \\
\hline Air bronchogram & $60(59.4 \%)$ \\
\hline Bronchial wall thickening & $62(61.4 \%)$ \\
\hline Fibrous strips & $28(27.7 \%)$ \\
\hline Nodular opacity with a surrounding halo & $15(31.5 \%)$ \\
\hline Pleural thickening & $12(11.9 \%)$ \\
\hline Pleural traction & $12(11.9 \%)$ \\
\hline Cavitation & $2(2.0 \%)$ \\
\hline Pleural effusions & $2(2.0 \%)$ \\
\hline Mediastinal lymphadenopathy & $0(0 \%)$ \\
\hline CT scores & $4.0 \pm 3.2$ \\
\hline
\end{tabular}




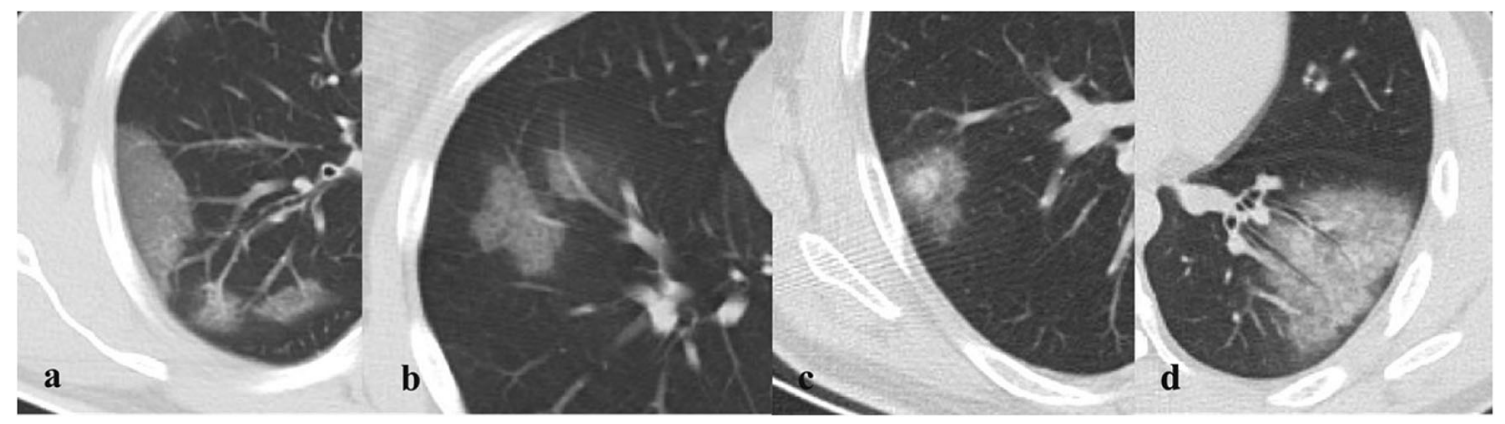

Fig. 1 Chest CT findings of COVID-19 pneumonia on axial images: (a) ground-glass opacities (GGO), (b) crazy-paving pattern, (c) nodular opacity with a surrounding halo, (d) air bronchogram and bronchial wall thickening

as mean \pm standard deviation (minimum-maximum) and the counting data were presented as the percentage of the total. CT pattern evolution analysis was performed according to the scatter diagram of each group, and the quadratic function was fitted with SPSS to obtain the corresponding curve. The comparisons of the quantitative data were evaluated using two-sample $t$ test, the comparative quantitative data of the same patient were evaluated using pair $t$ test, and counting
Table 3 Comparison between initial and first follow-up CT findings of 62 patients with COVID-19 infection

\begin{tabular}{|c|c|c|c|c|}
\hline Category & Initial chest $\mathrm{CT}$ & $\begin{array}{l}\text { First follow-up } \\
\text { CT }\end{array}$ & $\begin{array}{l}\text { Chi-square/Fisher's } \\
\text { exact } / t \text { test }\end{array}$ & $p$ value \\
\hline \multicolumn{3}{|l|}{ Unilateral or bilateral lung } & 11.513 & 0.002 \\
\hline No involvement & $5(8.1 \%)$ & $0(0 \%)$ & & \\
\hline Unilateral lung & $13(21.0 \%)$ & $4(6.5 \%)$ & & \\
\hline Bilateral lung & $44(71.0 \%)$ & $58(93.5 \%)$ & & \\
\hline \multicolumn{3}{|l|}{ Number of lobes affected } & 11.852 & 0.032 \\
\hline 0 & $5(8.1 \%)$ & $0(0 \%)$ & & \\
\hline 1 & $10(16.1 \%)$ & $3(4.8 \%)$ & & \\
\hline 2 & $7(11.3 \%)$ & $9(14.5 \%)$ & & \\
\hline 3 & $6(9.7 \%)$ & $7(11.3 \%)$ & & \\
\hline 4 & $10(16.1 \%)$ & $7(11.3 \%)$ & & \\
\hline 5 & $24(38.7 \%)$ & $36(58.1 \%)$ & & \\
\hline \multicolumn{3}{|l|}{ Density feature } & 21.84 & $<0.001$ \\
\hline No involvement & $5(8.1 \%)$ & $0(0 \%)$ & & \\
\hline Only GGO & $44(71.0 \%)$ & $27(43.5 \%)$ & & \\
\hline Only consolidation & $3(4.8 \%)$ & $2(3.2 \%)$ & & \\
\hline Both GGO and consolidation & $10(16.1 \%)$ & $33(53.2 \%)$ & & \\
\hline \multicolumn{3}{|l|}{ Distribution feature } & 2.17 & 0.558 \\
\hline Subpleural & $38(61.3 \%)$ & $39(62.9 \%)$ & & \\
\hline Random & $6(9.7 \%)$ & $5(8.1 \%)$ & & \\
\hline Peribronchovascular & $3(4.8 \%)$ & $8(12.9 \%)$ & & \\
\hline Subpleural and peribronchovascular & $10(16.1 \%)$ & $10(16.1 \%)$ & & \\
\hline \multicolumn{3}{|l|}{ Morphology } & l & / \\
\hline Nodular & $31(50.0 \%)$ & $15(24.2 \%)$ & & \\
\hline Lobar or segmental & $9(14.5 \%)$ & $9(14.5 \%)$ & & \\
\hline Patchy & $39(62.9 \%)$ & $47(75.8 \%)$ & & \\
\hline Irregular & $6(9.7 \%)$ & $33(53.2 \%)$ & & \\
\hline \multicolumn{3}{|l|}{ Margins } & 1.707 & 0.257 \\
\hline Ill-defined & $48(77.4 \%)$ & $57(91.9 \%)$ & & \\
\hline Well-defined & $9(14.5 \%)$ & $5(8.1 \%)$ & & \\
\hline \multicolumn{5}{|l|}{ Signs } \\
\hline Crazy-paving pattern & $22(35.5 \%)$ & $21(33.9 \%)$ & 0.036 & 1.000 \\
\hline Vascular enlargement & $21(33.9 \%)$ & $29(46.8 \%)$ & 2.145 & 0.2 \\
\hline Air bronchogram & $32(51.6 \%)$ & $39(62.9 \%)$ & 1.615 & 0.276 \\
\hline Bronchial wall thickening & $32(51.6 \%)$ & $45(72.6 \%)$ & 5.791 & 0.026 \\
\hline Fibrous strips & $8(12.9 \%)$ & $46(74.2 \%)$ & 47.369 & $<0.001$ \\
\hline Pleural thickening & $6(9.7 \%)$ & $23(37.1 \%)$ & 13.008 & 0.001 \\
\hline Pleural traction & $5(8.1 \%)$ & $13(21.0 \%)$ & 4.159 & 0.072 \\
\hline CT scores & $3.9 \pm 3.2$ & $6.2 \pm 3.8$ & -3.585 & $<0.001$ \\
\hline
\end{tabular}


data were evaluated using Chi-square test or Fisher's exact test. A $p$ value of $<0.05$ was defined as statistically significant.

\section{Results}

\section{Clinical characteristics}

A total of 110 subjects (67 males and 43 females) were included in the study (Table 1). Forty-eight patients had a history of sick contact with proven COVID-19 cases, 46 patients had a history of living in or traveling to Wuhan within 14 days before onset of symptoms, and the remaining 16 patients had unknown exposure history. The main presenting symptoms were fever $(92.7 \%)$ and cough (64.5\%). Up to the last day of follow-up CT (February 9, 2020), 32 of 110 patients were discharged in accordance with clinical cure standard.

\section{Chest CT image analysis}

All 110 patients underwent initial CT after admission per hospital protocol. The mean time from symptom onset to initial CT was 4.4 days (range from 0 to 15 days). For initial CT images in 110 patients, bilateral multiple subpleural GGO were the predominant pattern seen on initial chest CT (Table 2 and Fig. 1). Ill-defined patchy or nodular opacities were observed more frequently. Other CT presentations include crazy-paving, nodular opacity with surrounding halo, and air bronchograms with bronchial wall thickening (Fig. 1). The right lower lung $(86.1 \%)$ was most commonly involved. No mediastinal lymphadenopathy was present. Nine of $110(8.2 \%)$ patients had normal initial chest CT. The total lung severity scores ranged from 0 (in the nine normal $\mathrm{CT}$ ) to a maximum of 14 , with a mean scores of 4.0.

All of the 110 patients had a follow-up chest CT (second CT) during the study period. The mean time between initial and follow-up chest CT was 5.3 days (range 2-9 days). Six of 110 patients showed no interval changes; and of them, 4 had both normal initial and first follow-up CT. Sixty-two of 110 patients showed progression (Table 3): 20 patients showed density progression from GGO to consolidation, 17 patients showed increasing number of opacities, 8 patients showed overall progression with both opacities increasing and decreasing, 44 patients showed increase in extent of opacities. The mean CT scores of these 62 patients increased from 3.9 (range 0-14) to 6.2 (range 1-19). Forty-two patients demonstrated interval improvement; the mean CT scores of these 42 patients decreased from 4.5 (range 1-14) to 4.0 (range 1-14).

Sixty of the 110 patients had second follow-up chest CT (third CT) during the study period. The mean time between second and third chest $\mathrm{CT}$ was 4.4 days (range 2-8 days).
Three of 60 patients had no interval change; and of them, 2 had normal CT scans on their initial and first follow-up chest CT. Forty-five patients had interval improvement and 12 patients showed progression.

Using previously established stages of COVID-19 pneumonia found in Zhou et al, which included early rapid progressive stage (1-7 days), advanced plateau stage (814 days), and absorption stage (> 14 days) [14], we have identified four patterns of CT progression (Fig. 2). Type 1 pattern has relatively high initial CT scores with minimal progression and subsequent improvement. Type 2 pattern showed rapid progression of $\mathrm{CT}$ findings with subsequent CT improvement. Type 3 pattern was a static CT appearance with low CT scores throughout the imaging followup. Type 4 pattern was similar to type 2 but with a more prolonged course and more severe CT findings.

Type 1 pattern has mean CT scores of 5.27 and mean time to peak of 5.8 days since symptom onset. Type $1 \mathrm{CT}$ pattern was seen in $25 \%(15 / 60)$ of patients (sample case is shown on Fig. 3). Type 2 pattern has mean CT scores of 7.1 and mean time to peak of 10.7 days from initial symptom onset. Type 2 CT pattern was seen in $61.7 \%$ (37/60) of patients (sample case is shown on Fig. 4). Type 3 pattern was seen in $5.0 \%(3 / 60)$

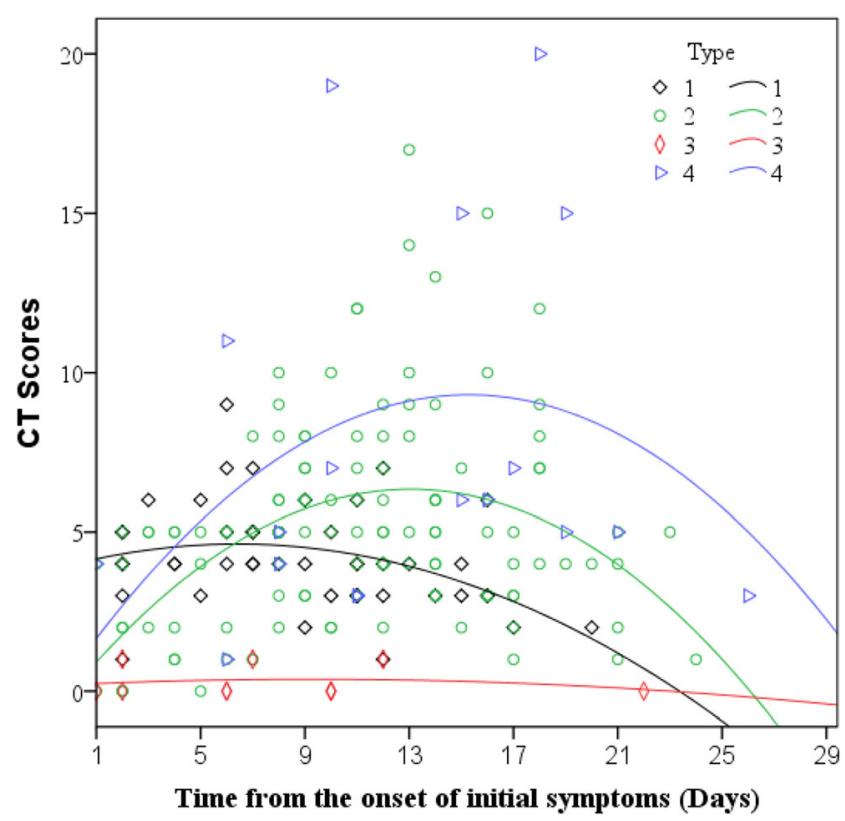

Fig. 2 Schematics depicting the four patterns of CT progression determined from three serial chest CT scans in 60 COVID-19 patients. Type 1: peak total CT lung involvement occurred within 7 days (curve fitting equation: $y=0.206 \times{ }^{2}-0.16 \mathrm{x}+3.968$ ); type 2 : peak total CT lung involvement occurred from days 8 to 14 (curve fitting equation: $y=$ $\left.0.975 \times{ }^{2}-0.037 \mathrm{x}-0.024\right)$; type 3 : peak total CT lung involvement occurred after day 14 (curve fitting equation: $y=0.037 \times{ }^{2}-0.002 \mathrm{x}+$ $0.202)$; type 4 : patients with asymptomatic infection or with CT scores less than 1 (curve fitting equation: $y=1.146 \times^{2}-0.038 x+0.548$ ), in which $x=$ time from the onset of the initial symptoms, $y=$ total CT scores of the pulmonary involvement). See Table 4 for time to peaks 

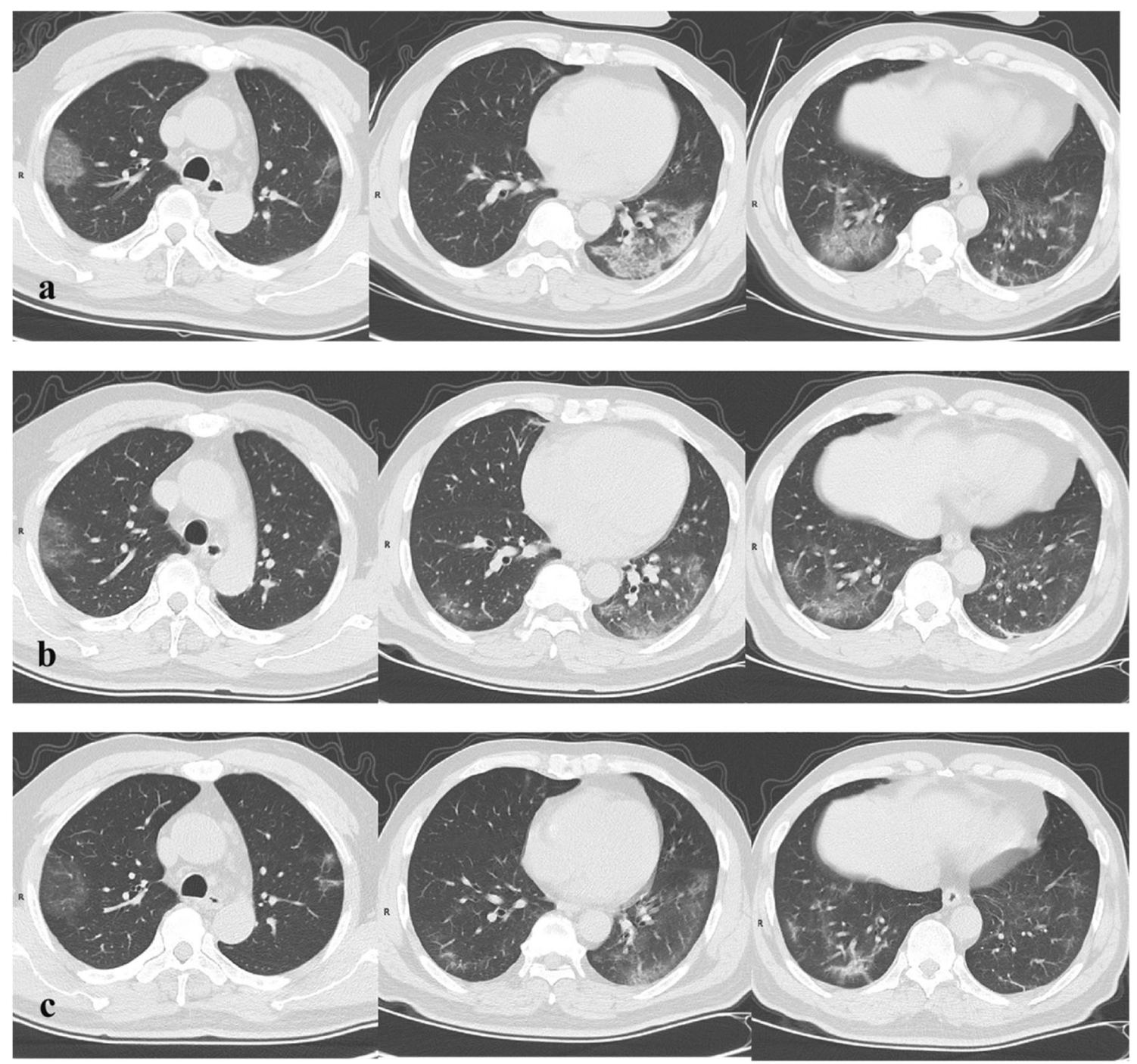

Fig. 3 Type 1 pattern. A 54-year-old male patient presenting with persistent fever for 6 days. a Initial axial thin-section CT images (day 6 after onset of symptom, January 28,2020 ) showed multifocal region of subpleural GGO with partial consolidation and irregular linear opacities in the bilateral lung, (b) follow-up CT on day 9 (January 31, 2020) showed reduced focal attenuation, (c) follow-up CT on day 14 (February 4, 2020) showed reduced focal attenuation with residual linear opacities patients. These patients had very mild clinical course. Type 4 pattern was similar to type 2 but with a more prolonged course and more severe CT findings. Average of CT scores was 10.8 with time to peak 17 days from onset of initial symptoms. Type 4 CT pattern was seen in $8.3 \%(5 / 60)$ of patients (a sample case is shown in Fig. 5). Time to peak for the four types of CT patterns is summarized in Table 4 .

The body temperatures of these 60 patients were shown in Table 5 which showed statistically significant difference in total days of fever in type 1 , type 2, type 3 , and type $4(p<0.05)$. There were significant differences in days of preadmission fever between type 1 and type $4(p<0.05)$. There was also statistically significant maximum body temperature in type 4 compared to type $3(p<0.05)$.
In addition, 17 of the 60 patients had a third follow-up CT (fourth CT) during the study period. The mean time between second and third follow-up CT was 5.0 days (range 3-8 days). Fifteen showed progression, one no interval change, and one interval improvement.

\section{Discussion}

The purpose of this study was to provide further data for imaging characteristics and to determine the pattern of change in chest $\mathrm{CT}$ associated with COVID-19 pneumonia from serial CT examinations. Our results showed that GGO were the most common pattern seen on initial chest CT. GGO with no consolidation occurred in $65.4 \%$ of patients which is 

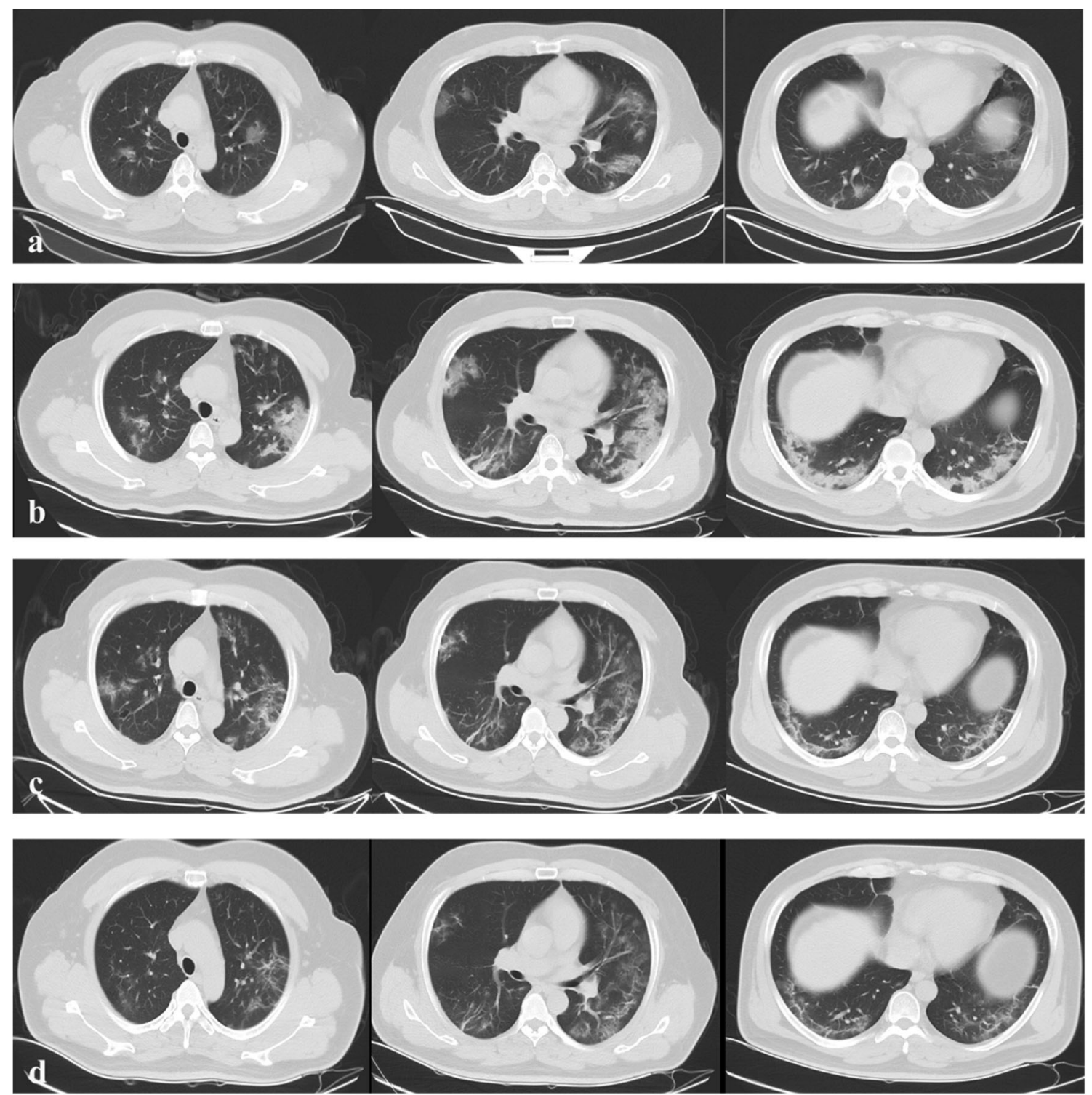

Fig. 4 Type 2 pattern. A 45-year-old male patient presenting with persistent fever for 4 days. a Initial axial thin-section CT images (day 5 after onset of symptom, January 21,2020) showed a small region of subpleural GGO in bilateral lung lobe, (b) follow-up CT on day 10 (January 26, 2020) showed increased number and extent of GGO in bilateral lung

similar to that of Chung et al, where 12 of 21 patients (57\%) had GGO only [7]. The subpleural location of opacities was another common CT feature of COVID-19 pneumonia in our study. The opacities occupied a subpleural or mixed subpleural and peribronchovascular location in $77 \%$ of patients. These findings are consistent with findings by $\mathrm{Fu}$ et al, which studied 56 laboratory-confirmed patients and showed that $32.7 \%$ (18) with GGO only, $52.7 \%$ (29) had GGO and consolidative opacities, and $14.5 \%$ (8) with only consolidation. A total of $54.5 \%$ of the opacities involved both peripheral and central parenchymas and $45.5 \%$ were purely peripheral distribution [13]. lobes, (c) follow-up CT on day 15 (January 31,2020 ) showed progression of disease with decreased extent and density of opacities, (d) follow-up CT on day 19 (February 4, 2020) showed reduced focal attenuation with residual linear opacities

Our study showed that the right lower lung $(86.1 \%)$ was most commonly involved which is also found by Zhou et al, which showed that the middle and lower zones were more commonly involved [14]. This pattern may be associated with the shorter right lower lobe bronchial anatomy. Pertinent negatives included the lack of mediastinal lymphadenopathy and pleural effusions.

First follow-up CT (approximately 9 days after onset of symptom) commonly showed progression of disease as manifested by increased extent and density of lung opacities. A combination of GGO and consolidation was the predominant pattern on the first follow-up chest CT. In this study, 60 

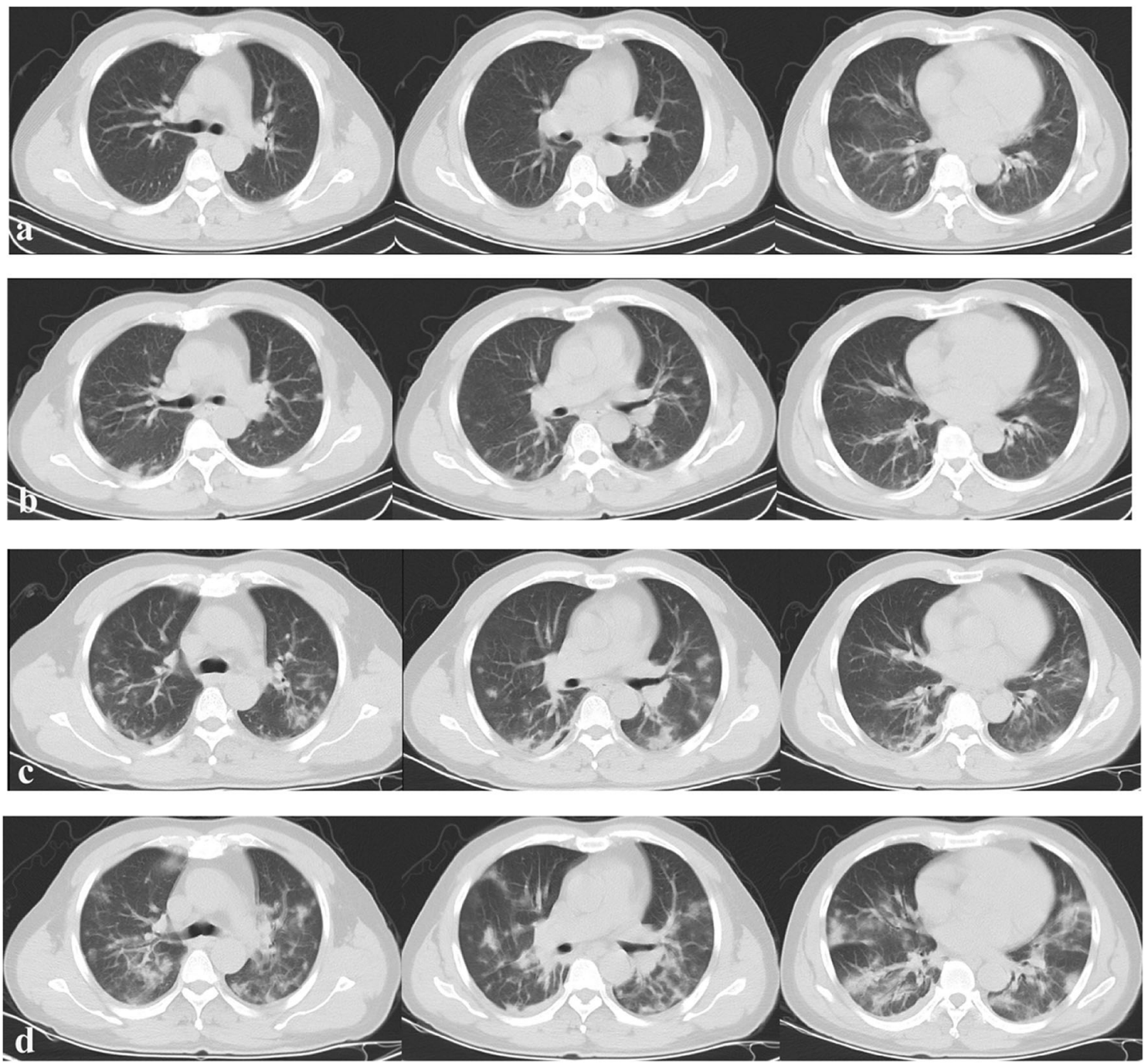

Fig. 5 Type 4 pattern. A 51-year-old male patient with fever for $4 \mathrm{~h}$. a Initial axial thin-section CT images (day 0 after onset of symptom, January 22, 2020) showed no involvement, (b) follow-up CT on day 4 (January 26, 2020) showed newly developed subpleural GGO in bilateral lung, (c) follow-up CT on day 8 (January 30, 2020) showed increase in extent of GGO with partial consolidation, (d) follow-up CT on day 12 (February 3, 2020) showed increase in extent of GGO with partial consolidation and superimposed inter- and intralobular septal thickening patients had second follow-up CT scans (approximately 13 days after onset of symptom) providing a more reliable data set for establishing the pattern of CT evolution. The most common CT pattern was type 2 in $61.7 \%$ of patients which showed progression of CT findings from initial to first follow- up CT scans with maximum difference in overall lung involvement. There is subsequent improvement on the second follow-up CT. Type 2 pattern is in accordance with a previous case report presenting the longitudinal changes of $\mathrm{CT}$ findings in COVID-19 pneumonia [15].
Table 4 Patterns of CT evolution of COVID-19 in 60 patients with three CT reexaminations

\begin{tabular}{lcclcrr}
\hline Progression pattern & Type 1 & Type 2 & Type 3 & Type 4 & $F$ value & $p$ value \\
\hline No. of cases & $15(25.0 \%)$ & $37(61.67 \%)$ & $3(5.0 \%)$ & $5(8.33 \%)$ & $/$ & $/$ \\
CT scores & $5.27 \pm 1.5$ & $7.1 \pm 3.0$ & N/A & $10.8 \pm 6.4$ & 6.065 & 0.004 \\
*Time to peak (days) & $5.8 \pm 1.3$ & $10.7 \pm 2.2$ & N/A & $17.0 \pm 1.5$ & 70.157 & $<0.001$ \\
\hline
\end{tabular}

Post hoc test (Bonferroni) showed there are significant differences between the type 1, type 2, and type 4 of time to peak, and significant differences between the type 1 and type 4 , and type 2 and type 4 of CT scores $(p<0.05)$. N/A not applicable. *Time to peak is defined as the time from the onset of initial symptom (in days) when the peak total CT scores were reached 
Table 5 Body temperature of 60 patients with COVID-19

\begin{tabular}{|c|c|c|c|c|c|c|}
\hline & Type 1 & Type 2 & Type 3 & Type 4 & $F$ value & $p$ value \\
\hline Days of fever prior to admission & $2.47 \pm 1.73$ & $3.77 \pm 3.30$ & $1.00 \pm 1.73$ & $6.27 \pm 3.61$ & 4.424 & 0.007 \\
\hline Days of fever in hospital & $4.20 \pm 2.98$ & $5.23 \pm 4.37$ & $2.67 \pm 4.62$ & $7.27 \pm 5.29$ & 1.491 & 0.227 \\
\hline Total days of fever & $6.67 \pm 3.58$ & $9.00 \pm 4.86$ & $3.67 \pm 4.04$ & $13.55 \pm 4.48$ & 6.567 & 0.001 \\
\hline The highest temperature & $37.91 \pm 0.74$ & $38.08 \pm 0.68$ & $36.93 \pm 0.15$ & $38.43 \pm 0.88$ & 3.611 & 0.019 \\
\hline
\end{tabular}

Post hoc test (Bonferroni) showed there are significant differences between the type 1, type 2, type 3, and type 4 of total days of fever, and significant differences between the type 1 and type 4 of days of fever prior to admission, and significant differences between type 3 and type 4 of the highest temperature $(p<0.05)$

On the initial CT (early stage, averaged 4 days after symptom onset), the CT showed mainly subpleural bilateral GGO. On the first follow-up CT (progression stage, averaged 9 days after symptom), the pattern develops into predominantly a mix pattern of GGO and consolidation. On the second follow-up CT (resolution stage, averaged 13 days after symptom onset), the pleural findings have started to resolve. These results were consistent with previously reported temporal lung changes on chest $\mathrm{CT}$ in patients with COVID-19 infection [10]. Zhou et al studied 100 patients with a total of $272 \mathrm{CT}$ scans and found that in the early rapid progressive stage (1-7 days), 58.1\% were GGO with reticular pattern, $43 \%$ GGO with consolidation, and $41.9 \%$ GGO only. In the advanced stage (8-14 days), $79.8 \%$ had GGO with consolidation. In the absorption stage, GGO plus consolidation decreased significantly [14].

There were significant differences in days of preadmission fever between the type 1 and type $4(p<0.05)$ and statistically significant maximum body temperature in type 3 compared to type $4(p<0.05)$. These findings provide a preliminary indication that $\mathrm{CT}$ patterns may be able to assist in predicting clinical course.

There are some limitations to our early study. The patients in this study were heterogeneous and while we were able to obtain the body temperature of the patients, further respiratory status data, such as oxygen requirement, blood oxygen saturation, and respiratory therapy requirements were not available. Future study will have to include more clinical symptom and CT pattern correlation. The different number of CT scans in the different groups could introduce selection bias as the patients with four serial CT scans could have more severe clinical symptoms compared with the groups with fewer serial CT scans. The differences in the number of patients in each $\mathrm{CT}$ pattern group also pose a statistical analytic challenge, especially the small sample numbers in type 3 and type 4 , which renders them underpowered and susceptible to statistical errors. Another limitation is the nonuniform scanning intervals among all patients due to the retrospective nature of this study.

In conclusion, the most common CT manifestation in hospitalized patients with COVID-19 infection is that of bilateral multiple peripheral GGO or consolidation. The most commonly shown evolution pattern was progression of disease with increased extent and density of lung opacities on the first follow-up CT (averaged 9 days after symptom onset) followed by improvement of disease on second follow-up CT (averaged 13 days after symptom onset). Preliminary findings suggest that CT pattern may be able to assist in predicting clinical course; future dedicated studies with clinical correlations will be needed.

Acknowledgments The authors would like to express their appreciation to the many members of the frontline medical staff for their selfless dedication and heroic dedication in the face of COVID-19 outbreak. We thank the editors and reviewers for their constructive criticisms and thoughtful comments on our manuscript.

Funding information This work was supported Emergency Science and Technology Project for COVID-19 of Jiangxi province (202011-2) (Wei Zhang).

\section{Compliance with ethical standards}

Guarantor The scientific guarantor of this publication is Dechang Peng.

Conflict of interest The authors of this manuscript declare no relationships with any companies whose products or services may be related to the subject matter of the article.

Statistics and biometry No complex statistical methods were necessary for this paper.

Informed consent Written informed consent was waived by the Institutional Review Board.

Ethical approval Institutional Review Board approval was obtained by the Human Research Ethics Committee of the First Affiliated Hospital of Nanchang University. The study performed according to the Declaration of Helsinki.
Methodology
- retrospective
- diagnostic or prognostic study
- performed at one institution

\section{References}

1. Huang C, Wang Y, Li X et al (2020) Clinical features of patients infected with 2019 novel coronavirus in Wuhan, China. Lancet 
395(10223):497-506. https://doi.org/10.1016/S0140-6736(20) 30183-5

2. Li Q, Guan X, Wu P et al (2020) Early transmission dynamics in Wuhan, China, of novel coronavirus-infected pneumonia. N Engl J Med 382(13):1199-1207. https://doi.org/10.1056/ NEJMoa2001316

3. Wang D, Hu B, Hu C et al (2020) Clinical characteristics of 138 hospitalized patients with 2019 novel coronavirus-infected pneumonia in Wuhan, China. JAMA 323(11):1061-1069. https://doi. org/10.1001/jama.2020.1585

4. Phan LT, Nguyen TV, Luong QC et al (2020) Importation and human-to-human transmission of a novel coronavirus in Vietnam. N Engl J Med 382(9):872-874. https://doi.org/10.1056/ NEJMc2001272

5. Chan JF, Yuan S, Kok K et al (2020) A familial cluster of pneumonia associated with the 2019 novel coronavirus indicating person-to-person transmission: a study of a family cluster. Lancet 395(10223):514-523. https://doi.org/10.1016/S0140-6736(20) 30154-9

6. WHO. https://www.who.int/emergencies/diseases/novelcoronavirus-2019/situation-reports/. Accessed 29 Apr 2020

7. Chung M, Bernheim A, Mei X et al (2020) CT imaging features of 2019 novel coronavirus (2019-nCoV). Radiology 295(1):202-207. https://doi.org/10.1148/radiol.2020200230

8. Liu P, Tan XZ (2020) 2019 novel coronavirus (2019-nCoV) pneumonia. Radiology 295(1):19. https://doi.org/10.1148/radiol. 2020200257

9. Fang Y, Zhang H, Xu Y, Xie J, Pang P, Ji W (2020) CT manifestations of two cases of 2019 novel coronavirus $(2019-\mathrm{nCoV})$ pneumonia. Radiology 295(1):208-209. https://doi.org/10.1148/ radiol.2020200280

10. Pan F, Ye T, Sun P et al (2020) Time course of lung changes at chest CT during recovery from coronavirus disease 2019 (COVID19). Radiology 295(3):715-721. https://doi.org/10.1148/radiol. 2020200370

11. Ooi GC, Khong PL, Muller NL et al (2004) Severe acute respiratory syndrome: temporal lung changes at thin-section $\mathrm{CT}$ in 30 patients. Radiology 230(3):836-844. https://doi.org/10.1148/radiol. 2303030853

12. Franquet $T$ (2011) Imaging of pulmonary viral pneumonia. Radiology 260:18-39. https://doi.org/10.1148/radiol.11092149

13. Fu F, Lou J, Xi D et al (2020) Chest computed tomography findings of coronavirus disease 2019 (COVID-19) pneumonia. Eur Radiol 1-10. https://doi.org/10.1007/s00330-020-06920-8

14. Zhou S, Zhu T, Wang Y, Xia L (2020) Imaging features and evolution on CT in 100 COVID-19 pneumonia patients in Wuhan, China. Eur Radiol 1-9. https://doi.org/10.1007/s00330-02006879-6

15. Wu Y, Xie Y, Wang X (2020) Longitudinal CT findings in COVID-19 pneumonia: case presenting organizing pneumonia pattern. Radiology: Cardiothoracic Imaging 2(1):e200031. https://doi. org/10.1148/ryct.2020200031

Publisher's note Springer Nature remains neutral with regard to jurisdictional claims in published maps and institutional affiliations. 